\title{
Intramolecular Radical formation-induced chemical stability of novel ZW-MOFs S. Alomari ${ }^{1}$ \\ ${ }^{1}$ Clarkson university \\ alomars@clarkson.edu
}

Metal-Organic Frameworks (MOFs) are hybrid crystalline porous materials composed of metal ions or clusters connected by polytopic organic linkers. MOFs are known for their enormous surface areas and large solventaccessible voids. Due to the infinite connection possibilities of their building blocks, MOFs have versatile topologies, which can be tailored to meet the demands for different applications such as small-molecule storage and separation, catalysis, and sensing. Incorporating zwitterionic (ZW) linkers in the frameworks can introduce charged organic surfaces in their cavities, leading to intermolecular electrostatic fields. Polarizable guest molecules can be attracted by these fields yielding enhanced guest-framework interactions. In this presentation, three isostructural novel zwitterionic metal-organic frameworks (ZW MOFs) materials $\{[\mathrm{Cd}(\mathrm{L}) 2] 1.5 \mathrm{DMF}\} \mathrm{n}$ MOF 1I,

$\{[\mathrm{Cd}(\mathrm{L}) 2] \cdot 1.5 \mathrm{DMF}\} \mathrm{n}$ MOF $1 \mathrm{II}$ and $\{[\mathrm{Zn}(\mathrm{L}) 2] \cdot 1.5 \mathrm{DMF}\} \mathrm{n}$ MOF 2 are reported. By the reaction of the rationally designed rigid anionic pyridinium-based zwitterionic ligand, 3-carboxy-1-(3-carboxybenzyl) pyridinium bromide $(\mathrm{H} 2 \mathrm{LBr})$ with $\mathrm{Cd}(\mathrm{ClO} 4) 2 \cdot 6 \mathrm{H} 2 \mathrm{O}$ and $\mathrm{Zn}(\mathrm{BF} 4) 2 \cdot \mathrm{H} 2 \mathrm{O}$. The two novel polymorphic Cd-based ZW MOFs forms, 1I and 1II, were identified during the crystallization experiment. The polymorph MOF 1I shows high stability in the air compared to $1 \mathrm{II}$ form and MOF 2. However, MOF 2 interestingly demonstrates better air stability upon ultraviolet (UV) irradiation exposure accompanied by a color change from yellow to dark brown. Both Electron paramagnetic resonance (EPR) analysis and temperature-dependent magnetic susceptibility data of MOF 2 after UV irradiation, provide conclusive proof for improving the stability due to radical formation. All these MOFs were structurally characterized by single-crystal X-ray diffraction (SCXRD), Electron paramagnetic resonance (EPR) analysis, infrared spectroscopy (IR), powder X-ray diffraction (PXRD) pattern, and adsorption measurements. 